\title{
Qualitative Data Archiving in Switzerland
}

\author{
by Brian Kleiner, Claudia Heinzmann, Thomas S. Eberle, Manfred \\ Max Bergman'
}

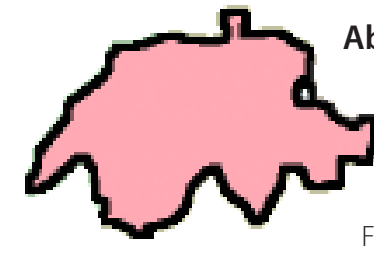

Abstract

At the time of the Bremen workshop in 2009, there

was no Swiss institution responsible for archiv-

ing qualitative social science data collected in

Switzerland. Since that time, the Swiss institution

FORS (Swiss Centre of Expertise in the Social Sciences) has assumed this role, with development of the infrastructure, policy, and know-how needed for implementation. The archiving of qualitative data at FORS is now moving forward in close collaboration with Swiss universities with established and strong qualitative research traditions. Over time, the success of qualitative data archiving at FORS will require the support of research funding institutions and policymakers, enhanced educational initiatives at universities, promotion of the value and potential of secondary data analysis, and a well-equipped and staffed archive that serves also as a network node and resource centre.

Keywords: Secondary use; archives; data; qualitative research; research infrastructure; Switzerland

\section{Introduction}

During the last two decades, qualitative inquiries have gained increasing popularity among European researchers in the social sciences and related fields. However, despite the institutionalization and legitimization of qualitative inquiries, this form of research "has not yet reached the same significance and reputation in Switzerland as it has in many other countries" (Eberle \& Bergman 2005: 1). Qualitative research in Switzerland is "lagging behind with regard to networks and structures that could offer information, support, resources, quality control and advanced training" (Eberle 2005: 4). Whilst there exists federal, cantonal, and private archives in Switzerland that provide access to a variety of historical data, at the time of the Bremen workshop in 2009 there was still no Swiss archive for data collected within the framework of qualitative research projects in the social and related sciences. Neither was there a resource centre or formalized network for offering services, information, and advice for researchers working within the qualitative research tradition. Since that time, FORS (Swiss Centre of Expertise in the Social Sciences) has assumed the role of central archive for qualitative data produced in Switzerland, and in coordination with various institutions and researchers has begun to make available resources for qualitative work.

In this article we describe some of the potential for and obstacles to qualitative data archiving in Switzerland. Since archiving and re-use of qualitative data has to be discussed within a wider framework of quality concerns of qualitative inquiries (Bergman \& Coxon 2005; Eberle 2005), we first describe recent steps in promoting qualitative research in Switzerland. We then examine current challenges to maintaining a qualitative data archive, and close by discussing future prospects in Switzerland.

\section{The current sitution in Switzerland: steps toward promoting qualitative research and data archiving}

As a result of the contrast between the increasing numbers of qualitative studies on the one hand and the lack of institutionalization of qualitative research on the other, the Swiss Academy for Humanities and Social Sciences (SAGW/ASSH) has launched several initiatives to promote qualitative research in Switzerland ${ }^{2}$. The primary goals of these initiatives are to build and strengthen networks, work toward best practices in methods and teaching, and to assess the feasibility of an archive and resource centre for qualitative research.

In cooperation with the former Swiss Information and Data Archive Service for the Social Sciences (SIDOS, now part of FORS, see below) and the Social Science Policy Council (a committee of the SAGW), a workshop was conducted in 2002 to identify the experiences of active qualitative researchers in Switzerland as well as key representatives of qualitative archives and similar institutions from other European countries ${ }^{3}$. This event led to subsequent meetings and to three working groups, which were asked by the SAGW to produce an informative and accessible document on (a) the possibilities and limits of qualitative research for the social and related sciences, (b) 
quality criteria for assessing research results from qualitative research, and (c) recommendations on how to integrate qualitative research methods into the university curriculum. A document summarising the issues elaborated by the working groups was discussed at a final meeting with international experts, found strong support from the qualitative research community in Switzerland, and a fully elaborated "statement" was published in 2009 (Bergman et al).

Since the 2009 Bremen workshop, FORS has taken concrete steps to establish the capacity for archiving qualitative data, including a workshop of archiving and research experts in 2010, development of specific policies and procedures, as well as workflow and system adjustments to integrate qualitative data into its database. With the capacity now in place, FORS is poised to introduce qualitative research data into its holdings.

\section{Obstacles to qualitative data archiving in Switzerland} While FORS has begun archiving data from qualitative research projects, it is not clear yet that the research community in Switzerland will take notice, deposit their data, and make use of the qualitative data of others. Specifically, there remain a variety of significant potential barriers:

1. The use of secondary data is considered to be more easily applicable for quantitative research than for qualitative studies. In general, qualitative researchers in Switzerland are not familiar with the possibilities of secondary data analysis, and they do not know which research topics or potentially available data sets are suitable for secondary data analysis. More specifically, the idea of developing research questions based on the data of "someone else" seems challenging. This difficulty is related to the belief that it is necessary for qualitative researchers to go through the whole process of data collection in order to contextualize the material in an appropriate way (e.g., Corti 2000: 26 for more details; for a critique, see Moore 2007).

2. The various types of qualitative data make it difficult to decide which material should be archived and provided for re-use. Among researchers, specific concerns arise in relation to supplementary materials, such as researchers' field notes and personal notes made before and after interviews. These materials are quite important in providing an interpretive context, but they are often considered to be too private or sensitive to be shared with other researchers. 3. One of the key concerns is related to the ethical and legal implications of rendering qualitative data accessible. That is, there is the problem of ensuring anonymity, confidentiality, and data protection. Can qualitative data be adequately and consistently anonymised without reducing the value of and interest in the data for re-use? If not, are there other ways to address adequately the need to protect confidentiality, such as informed consent or strict access conditions? Beyond questions of anonymization and data integrity, it is not entirely clear how Swiss law treats the subject of data protection. Although there are federal and cantonal laws on archiving and data protection (e.g. Confédération Suisse 2008; Grand Conseil du Canton du Vaud 2007), there are no national policies specifically relating to qualitative data. These legal issues should be addressed by specialists knowledgeable about Swiss law. 4. The various types of qualitative data (e.g. transcripts, field notes, audio and videotapes, pictures, etc.) present difficulties with respect to adequately preserving the data over a long period of time (see Corti 2000: 20f for a detailed discussion). FORS should have sufficient resources for ensuring the requisite infrastructure, staff, and knowhow for dealing with different and changing formats over time.
5. Finally, there are financial challenges in developing and maintaining an archive for qualitative data indefinitely.

In addition to these potential challenges, there is the problem of whether or not there will be significant interest in archived qualitative data. In Switzerland, data re-use is not a deeply engrained part of the research culture. Furthermore, secondary analysis is far more common with quantitative data. With respect to re-use and sharing of qualitative data, this happens only occasionally for individual projects. It is quite likely that the lack of work in this area is due to insufficient know-how and training on the part of most researchers, lack of available national data for re-use, a comparatively generous funding infrastructure for the collection of new data, and a general lack of awareness and appreciation about the value and potential of the re-use of high-quality data. It is clear that much work needs to be done to develop, advance, and promote re-use of qualitative data in Switzerland, which should include training, networking, and support in research grant applications.

\section{Development planning}

Currently, FORS is strongly interested in continuing in the direction of qualitative data archiving, dissemination, and the provision of additional resources to researchers. It has developed a set of institutional policies and procedures regarding qualitative data, as well as a guide to assist researchers in how to prepare their data for deposit. The social science research holdings within the archive at FORS are currently mostly quantitative, but now include data from several qualitative research projects. In concert with researchers within Switzerland (including several authors of this paper), future efforts will be devoted to promoting and encouraging secondary analyses of qualitative data and deposit of project data at FORS.

There are some gaps that should be noted. Realising qualitative archiving at FORS in the long-run will certainly require additional resources, including at least one new staff member and additional technical infrastructure and development. In any case, the continued development of qualitative data archiving in Switzerland should be done in close collaboration with experienced researchers and with universities where qualitative research is firmly established.

\section{Conclusion}

Even in quantitative research, secondary data analysis is not well established among some research branches in the social and related sciences in Switzerland. Nevertheless, most stakeholders in the social science research domain in Switzerland would agree on the various scientific and cost-benefit advantages of secondary data analysis of high-quality data from qualitative research projects. Realising a sophisticated data archive for qualitative research in Switzerland will have to include (a) the support of research funding institutions and research policy makers, (b) more systematic training in qualitative research at universities and the connected possibility of secondary data analysis, (c) a well-equipped and staffed archive that actively conducts outreach projects and serves as a network node and resource centre, and (d) the uptake of the use of an archive by the research community, encouraged and supported by funding bodies, an active research network, and their own methodological expertise.

\section{References}

Bergman, M.M \& Coxon, A.P.M. (2005).' The quality in qualitative methods. Forum Qualitative Sozialforschung / Forum: Qualitative Social Research. 6 (2). Art. 34

Bergman, M.M \& Eberle, T.S. (eds.). (2005). 'Qualitative inquiry: research, archiving, and reuse'. Forum Qualitative Sozialforschung / Forum: Qualitative Social Research. 6 (2) [Online]. Available at: (http://www. 
qualitative-research.net/index.php/fqs/issue/view/12). [Accessed 17th July 2009]

Bergman, M, Eberle, T., Flick, U, Förster, T, Horber E, Maeder, C, Mottier, V, Nadai, E, Rolshoven, J, Seale, C, Widmer, J. (2009). A statement on the meaning, quality assessment, and teaching of qualitative research methods.[Online]Available at. http://www.qualitativeresearch.ch/docs/Manifest_Qualitative_Sozialforschung_online.pdf

Confédération Suisse (2008). Loi fédérale sur l'archivage . [Online]. Available at : http://www.admin.ch/ch/f/rs/152_1/index.html [Accessed 17th July 2009]

Corti, L, Witzel, A \& Bishop, L (eds.) (2005). Secondary analysis of qualitative data, Forum Qualitative Sozialforschung / Forum: Qualitative Social Research. 6 (1). [online]Available at: http://www.qualitativeresearch.net/index.php/fqs/issue/view/13 [Accessed 17 July 2009]

Corti, L, Kluge, S, Mruck, K \& Opitz, D (eds.) (2000). 'Text, archive, reanalysis'. Forum Qualitative Sozialforschung / Forum: Qualitative Social Research,.[online] 1 (3) Available at:http://www.qualitativeresearch.net/index.php/fqs/issue/view/27>Accessed 17 July 2009]

Corti, L. (2000). 'Progress and problems of preserving and providing access to qualitative data for social research. The international picture of an emerging culture'. Forum Qualitative Sozialforschung / Forum: Qualitative Social Research. 1 (3). Art. 2

Eberle, T. (2005).'Promoting qualitative research in Switzerland'. Forum Qualitative Sozialforschung / Forum: Qualitative Social Research. 6 (2). Art. 31

Eberle, T.S. \& Bergman, M.M. (2005).' Introduction'. Forum Qualitative Sozialforschung / Forum: Qualitative Social Research. 6 (2) .Art. 30

Fielding, N.( 2005).'The resurgence, legitimation and institutionalization of qualitative methods' Forum Qualitative Sozialforschung / Forum: Qualitative Social Research. 6 (2). Art. 32

Le Grand Conseil du Canton du Vaud .(2007). Loi sur la protection des données personnelles. [Online]. Available at: http://www.rsv.vd.ch [Accessed 17th July 2009]

Moore, N.( 2007).' (Re)Using Qualitative Data?' Sociological Research Online. 12 (3).

\section{Notes}

1. Brian Kleiner FORS, Swiss Centre of Expertise in the Social Sciences, Lausanne, Switzerland

Claudia Heinzmann Institute of Sociology, University of Basel, Switzerland

Thomas S. Eberle Institute of Sociology, University of St. Gall, Switzerland

Manfred Max Bergman Institute of Sociology, University of Basel, Switzerland

Contact: Brian Kleiner, Brian.Kleiner@fors.unil.ch

2. For detailed discussions and reasons on why to promote qualitative research, see Eberle (2005).

3. Updated versions of the conference presentations are published in Bergman \& Eberle (2005). 